\title{
Modification of the Measurement Setup for Examination of Acoustic Field Distribution Inside Cylindrical Ducts for Axisymmetric Excitation
}

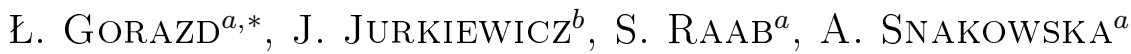 \\ AGH - University of Science and Technology \\ ${ }^{a}$ Faculty of Mechanical Engineering and Robotics \\ ${ }^{b}$ Faculty of Electrical Engineering, Automatics, Computer Science and Biomedical Engineering \\ al. A. Mickiewicza 30, 30-059 Krakow, Poland
}

\begin{abstract}
The aim of the paper is to present results of examination of the acoustic pressure field inside a model of a cylindrical duct. Measurements of the root mean square acoustic pressure were carried out using a new measurement setup, first in conditions allowing for propagation of the plane wave only, and next - by increasing the excitation frequency - with successive higher modes also taken in account. Two types of hard-walled cylindrical ducts were examined corresponding to mathematical models known as the infinite duct and the semi-infinite duct. A dedicated measurement setup is presented as well as the successive stages of its modification. In order to verify the modified measuring system, the first stage of the research work consisted in excitation of the axisymmetric modes only and assessing the degree of symmetry represented by the measurement data Significant improvement of the compatibility of the obtained measurement results with theoretical predictions was observed in the explored frequency band $800-5500 \mathrm{~Hz}$ that for the duct inside radius of $a=0.077 \mathrm{~m}$ corresponded to the reduced frequency range $1.13<k a<7.76$.
\end{abstract}

DOI: $10.12693 /$ APhysPolA.123.1085

PACS: 43.20.Rz, 43.20.Mv

\section{Introduction}

Theoretical analysis of the acoustic field inside perfectly hard-walled ducts predicts that, apart from the plane wave, they may carry wave modes of higher orders, known as Bessel modes in the case of cylindrical ducts. If the excitation frequency is higher than the so-called cutoff frequency for modes of a specific order, such modes can propagate as travelling (not attenuated) waves, otherwise they decay exponentially with distance [1].

Literature of the subject can be classified into three categories as far as the adopted accuracy of description of the related phenomena is concerned, of which two propose significant simplifications, namely the plane wave approximation [2] and the single mode approximation $[3,4]$. The third approach, the exact one, assumes multimodal nature of the propagating wave $[5,6]$.

The plane wave approximation is typically used in analyses concerning phenomena occurring in pipe-shaped elements of acoustic filters as well as in the whole class of problems related to tests carried out with the use of the impedance tube method [7] where elimination of higher modes and reduction of wave content to the plane wave only by proper choice of the excitation frequency that must be lower than the cut-off frequency for the first Bessel mode is more than desirable.

*corresponding author; e-mail: gorazd@agh.edu.pl
The single-mode approximation adopted usually in studies focused on the effect of diffraction at the duct outlet $[3,4]$ allows to understand the involved processes including, in the first place, the phenomenon of transformation of modes at the outlet $[3,4]$. However, by assuming the multimodal nature of the wave progressing towards the outlet it is possible to analyze all diffraction/reflection/transformation phenomena occurring in acoustic waveguides $[5,6,8]$ and obtain satisfactory consistence of the theoretical model with results of measurements; this applies in particular to wave guides with large radii such as e.g. shells of aircraft jet engines or ducts of heating, ventilation, and air conditioning (HVAC) systems $[9,10]$, in which additionally flow of the medium is often considered [11-13].

This paper presents results of examination of the acoustic pressure field inside a model of cylindrical duct, first in conditions allowing for propagation of plane wave only, and next - by increasing the excitation frequency - with successive higher Bessel modes also taken in account.

A dedicated measurement setup is also presented and successive stages of its modification with special emphasis put on microphone positioning accuracy necessary to observe consistency of measurement results with predictions of the exact theory.

\section{Mathematical models and their model embodiments}

Two types of hard-walled cylindrical ducts were examined in the framework of the present research work corre- 
sponding to mathematical models known as the infinite duct and the semi-infinite duct.

The concept of the infinite hard-walled duct refers to a waveguide on surface of which the normal component of the acoustic velocity is zero, with a sound source located very far from the observation point and producing a progressing wave propagating in one direction with no waves reflected back. A physical model of such structure can be obtained by closing a duct of finite length with a material intensively absorbing acoustic waves, i.e. providing a model duct with the so-called anechoic termination (Fig. 1a).

Further, the hard-walled semi-infinite duct is defined as a structure on surface of which the acoustic velocity normal component is zero that carries both wave modes progressing towards the open end and modes travelling backwards generated at the open end as a result of diffraction (Fig. 1b). According to theoretical results accounting for diffraction phenomena occurring at the outlet, acoustic wave in a duct of that type represents a sum of modes propagating towards the outlet, reflected from the outlet, and transformed at the outlet $[8,14]$.
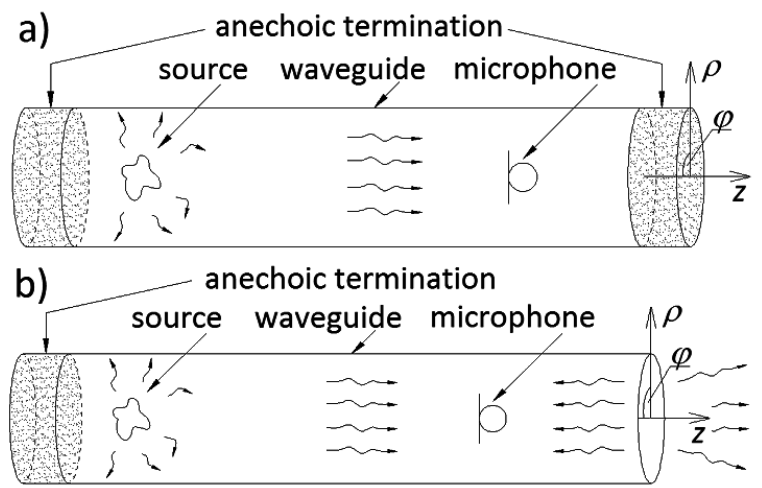

Fig. 1. Physical embodiments of mathematical concepts of: (a) the infinite duct; (b) the semi-infinite duct.

\section{Theoretical foundations}

The acoustic field inside a duct can be described by means of either the acoustic pressure field $p(\boldsymbol{r}, t)$ or the acoustic velocity field $\boldsymbol{v}(\boldsymbol{r}, t)$. Assuming linearity of the involved phenomena, with the use of Euler equation and the continuity equation one obtains the wave equation [1]:

$$
\Delta p(\boldsymbol{r}, t)-\frac{1}{c^{2}} \frac{\partial^{2} p(\boldsymbol{r}, t)}{\partial t^{2}}=0
$$

which for harmonic excitation assumed throughout the present paper in the form $\exp (-i \omega t)$ can be reduced to the Helmoltz equation [1]

$$
\Delta p(\boldsymbol{r}, t)+k^{2} p(\boldsymbol{r}, t)=0,
$$

where $k=\omega / c$ is the wave number.

By applying the boundary condition expressing rigidity of duct walls, i.e. $\left.v_{n}\right|_{\Sigma}=0$ where $v_{n}$ is the acoustic velocity normal component and $\Sigma$ is the duct surface, and with the $z$ coordinate axis coinciding with the duct's symmetry axis, the acoustic pressure of a single incident wave mode for an excitation characterized by the pulsatation $\omega$ can be expressed in the cylindrical coordinates by means of formula

$$
p_{m n}^{\mathrm{inc}}(\boldsymbol{r}, t)=A_{m n} e^{\mathrm{i} m \phi} J_{m}\left(\frac{\mu_{m n} \rho}{a}\right) e^{\mathrm{i}\left(\gamma_{m n} z-\omega t\right)},
$$

where $\mathrm{i}^{2}=-1, \gamma_{m n}=\sqrt{k^{2}-\frac{\mu_{m n}^{2}}{a^{2}}}=\frac{1}{a} \sqrt{(k a)^{2}-\mu_{m n}^{2}}$ is the longitudinal wave number, $m=0,1,2, \ldots, n=$ $1,2, \ldots$, are the indices of circumferential and radial mode order, $a$ is the duct radius, $J_{m}$ is the Bessel function of the first kind of order $m, \mu_{m l} / a$ is the transverse wave number, $\mu_{m l}$ is the $l$-th root of first derivative of the Bessel function $J_{m}$, i.e. the $l$-th solution of equation $J_{m} \prime\left(\mu_{m l}\right)=0$.

If the longitudinal wave number $\gamma_{m n}$ is a real number, then the corresponding mode can propagate in the duct without attenuation as a travelling wave and for that reason it is called the allowed mode (or the cut-on mode). For non-decaying modes of that type, condition $k a>\mu_{m l}$ must be met where the product $k a$ is called the reduced frequency or the Helmholtz number. Modes with imaginary longitudinal wave numbers $\left(k a<\mu_{m l}\right)$ called the cut-off modes represent acoustic field features decaying exponentially with distance from the source. If the longitudinal wave number $\gamma_{m n}$ is a real number, then the corresponding mode can propagate in the duct without attenuation as a travelling wave and for that reason it is called the allowed mode (or the cut-on mode). For non-decaying modes of that type, condition $k a>\mu_{m l}$ must be met where the product $k a$ is called the reduced frequency or the Helmholtz number. Modes with imaginary longitudinal wave numbers $\left(k a<\mu_{m l}\right)$ called the cut-off modes represent acoustic field features decaying exponentially with distance from the source.

In a semi-infinite duct, acoustic wave coming from the inside is subject to partial reflection from the open end resulting in interference with incoming modes and creation of standing waves. Apart from the reflection, also the effect of transformation of the incident modes into different ones (coupling) occurs according to the principle of conservation of the circumferential mode order $m$ [15]. Reflection and transformation coefficients describing quantitatively the phenomenon are complex number given either in the form of modulus and phase [15] or as the modulus and the so-called open-end correction [16].

Any steady-state acoustic wave observed in a semi-infinite circular duct is therefore, as a result of diffraction occurring at the unbaffled open end, a sum of all possible modes, even in the case when the wave incident at the duct end is a single mode. Hence, the formula representing the resulting acoustic field inside the duct must be adopted in the form $[15,16]$ :

$$
\begin{gathered}
p_{m n}(\rho, \phi, z)=A_{m n} e^{\mathrm{i} m \phi}\left[\frac{J_{m}\left(\mu_{m n} \frac{\rho}{a}\right)}{J_{m}\left(\mu_{m n}\right)} e^{\mathrm{i} \gamma_{m n} z}\right. \\
\left.+\sum_{n=1 / 0}^{N_{m}} R_{m l n} \frac{J_{m}\left(\mu_{m l} \frac{\rho}{a}\right)}{J_{m}\left(\mu_{m l}\right)} e^{-\mathrm{i} \gamma_{m l} z}\right]
\end{gathered}
$$


where $R_{m l n}$ is the coefficient describing transformation/reflection of the incident mode $(m, n)$ into mode $(m, l)$. The above formula accounts only for modes propagating from the duct outlet as non-decaying travelling waves, i.e. the cut-on modes, whereas $N_{m}$ denotes the maximum value of index $n$ for which the condition $k a>\mu_{m n}$ is met. It should be, however, noted that at the outlet itself $(z=0)$, contribution of the transformed cut-off modes to the resultant acoustic pressure may prove not negligible.

In practice, one can limit the analysis to the incident wave comprising only the cut-on modes also if multimodal excitation is considered, especially when the field far from the excitation source is the subject of interest. In such case, the total acoustic pressure $p^{\text {rmtot }}$ inside the duct will be the sum of components represented by cuton modes for the duct of given type at a given frequency,

$$
p^{r m t o t}=\sum_{m, n}^{p_{m n}},
$$

where summation is performed only over such pairs of indices for which $k a>\mu_{m n}$.

\section{The measurement setup}

Theoretical analysis of the acoustic field distribution inside cylindrical ducts has proved that for some types of sources one should expect very sudden variations of the acoustic pressure along the duct. The effect actually observed and shown in Fig. 2. In the tests carried out with laboratory models, this means the increased sensitivity of the measurement system to significant errors related to inaccuracy in positioning of the microphone and/or the sound source.

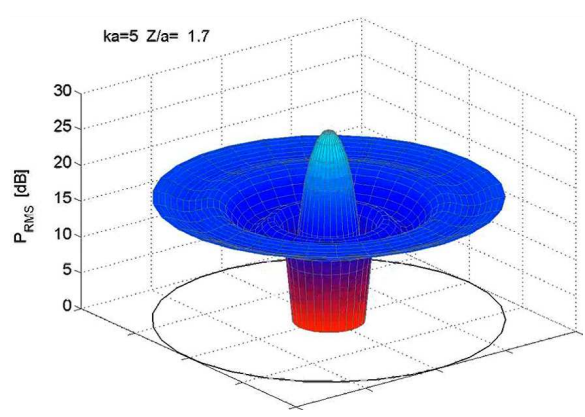

Fig. 2. The sudden variations in acoustic pressure along the duct radius predicted by means of numerical calculations.

The preliminary test measurements with the use of axisymmetric excitation proved a significant departure from axial symmetry indicating that a modification of the measurement setup was required. As a result of a thorough analysis of possible error sources it was decided that in modification of the system, special emphasis should be put on automation of the measurement process and precision of positioning individual components of the measuring circuit.

The most important new component of the setup, introduction of which was expected to increase significantly consistence of measurement results with predictions of the theoretical model, was a specially designed movable microphone-carrying head mounted at the end of the measuring arm (Fig. 3).

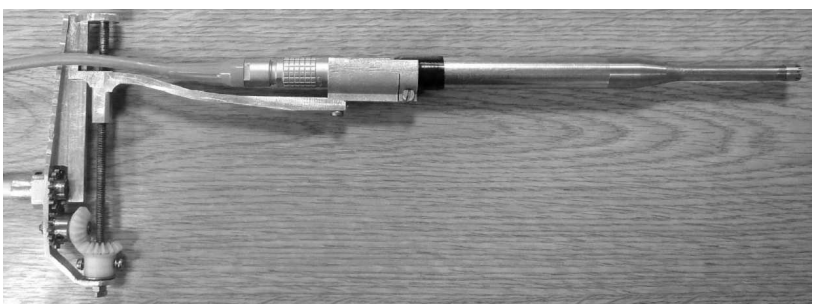

Fig. 3. The measuring head with $1 / 4$ " microphone.

Linear displacement of microphone within the head was obtained by transmission of rotational motion of a threaded pin with diameter of $4 \mathrm{~mm}$ engaged with guides restricting its motion in other directions. The use of the threaded pin allowed to position the microphone along the duct radius with high accuracy thanks to large gear ratio of the transmission. The drive element was a small computer-controlled stepping motor.

All elements of the measuring system are shown in a schematic diagram (Fig. 4). The measuring arm is provided with a small stabilizer the function of which is to compensate unavoidable deflection of the arm and position it precisely in the duct axis. The measuring arm was connected with computer-controlled AF-01 turntable and could be rotated by an arbitrary predetermined angle with $0.01^{\circ}$ accuracy. Such configuration ensured that the measuring head with microphone could be precisely positioned over the whole cross-section of the duct, i.e. at any point defined by cylindrical coordinates $\rho$ and $\varphi$. In order to take measurements, the head together with the measuring arm needed to be introduced into the duct, therefore in order to minimize the relating acoustic field disturbance, dimensions of the components were reduced to a minimum, including the use of a $1 / 4^{\prime \prime}$ microphone. AF-01 turntable together with the stepping motor was located horizontally on a wooden platform with adjustable height and could be moved forward and back on aluminum guides, that in turn allowed to move the microphone from one cross-section of the duct to another, i.e. along the $z$ axis of the system.

The whole measurement process has been automated. A dedicated code was developed in the MATLAB programming environment to control precisely position of the microphone and acquire the acoustic pressure measurement data.

In order to verify the measuring system modified this way, the first stage of the study consisted of observing axisymmetric modes and assessing the degree of symmetry represented by the measurement results. For that reason, a small wide-band loudspeaker located centrally on the duct axis was used as the sound source. Measurements were taken for the two duct models shown in Fig. 1. In 


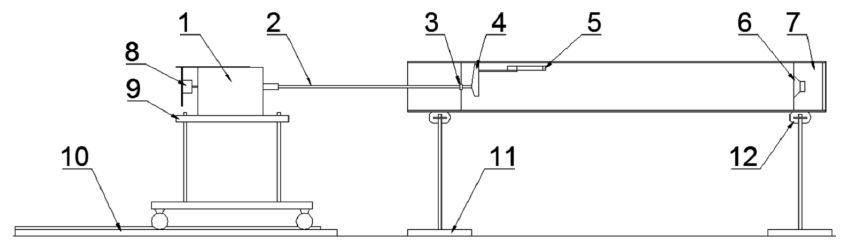

Fig. 4. Schematic diagram of the measurement setup: 1 - AF-01 turntable; 2 - measuring arm; 3 - measuring arm stabilizer; 4 - measuring head; 5 - measuring microphone; 6 - wide-band loudspeaker; 7 anechoic termination; 8 - stepper motor; 9 - platform; 10 - platform guides; 11 - duct supports, 12 vibro-insulation.

both cases, the gap between the loudspeaker and the duct wall (perimeter) was filled with $0.10 \mathrm{~m}$ thick layer of mineral wool. The wool pad together with the loudspeaker was closed with a round wooden cover making the socalled anechoic termination. In the case of measurements with the infinite duct model, the other duct end was also provided with analogous anechoic termination (wooden cover and a $0.10 \mathrm{~m}$ thick pad of mineral wool). For the tests requirements, a segment of circular plastic pipe was used inner radius of which was $a=0.077 \mathrm{~m}$ and length $l=2 \mathrm{~m}$.

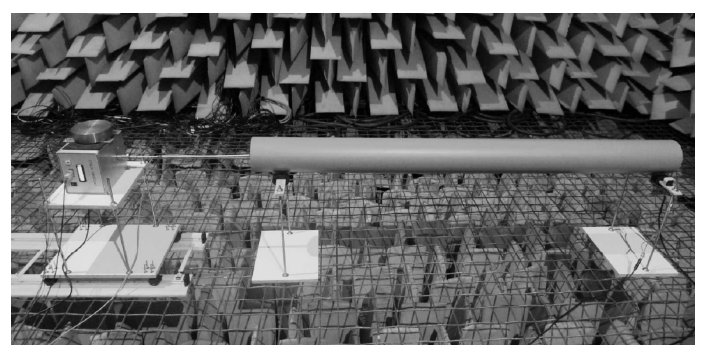

Fig. 5. Overall view of the measurement setup.

Signal frequencies used for testing the measurement setup were selected so that each interval between two consecutive test frequencies included the cut-on frequency for one new mode only. This allowed to observe changes occurring in the acoustic pressure pattern related to appearance of a consecutive Bessel mode predicted by the theory in progressing wave generated by the source. The frequencies for which the measurements were carried out (and the corresponding values of the reduced frequency) were adopted as follows: $800 \mathrm{~Hz}(k a \approx 1.13) ; 1730 \mathrm{~Hz}$ $(k a \approx 2.44) ; 2440 \mathrm{~Hz}(k a \approx 3.44) ; 2850 \mathrm{~Hz}(k a \approx 4.02)$; and $5500 \mathrm{~Hz}(k a \approx 7.76)$. Table lists the indices $(m, n)$ of all modes, both radial and circumferential, cut-on frequencies of which are lower than the selected $k a$ values.

Departure from axial symmetry observed in measurements could speak for excitation of non-axisymmetric allowable (cut-on) modes listed in the above table and, indirectly, for certain disturbances in the system's symmetry.
TABLE

Indices of the cut-on modes versus the reduced frequency ka.

\begin{tabular}{c|c|l}
\hline \hline$k a$ & No. of modes & \multicolumn{1}{c}{ Allowable modes } \\
\hline 1.13 & 1 & $(0,0)$ \\
2.44 & 2 & $(0,0)+(1,1)$ \\
3.44 & 3 & $(0,0)+(1,1)+(2,1)$ \\
4.02 & 4 & $(0,0)+(1,1)+(2,1)+(0,1)$ \\
7.76 & 11 & $(0,0)+(1,1)+(2,1)+(0,1)+(3,1)+(4,1)$ \\
& & \\
& &
\end{tabular}

The measurements were taken on the duct axis and at points located on thirteen concentric circles symmetric with respect to the duct axis separated by $5 \mathrm{~mm}$ and in $15^{\circ}$ increments of the azimuthal angle, over three different duct cross-section planes. Acoustic pressure values were registered by means of the Bruel\&Kjær 4135 $1 / 4^{\prime \prime}$ microphone. The measurements were carried out in free-field conditions, in the large anechoic chamber of the Department of Mechanics and Vibroacoustics, AGH - University of Science and Technology in Cracow

\section{Results of the measurements}

The following figures present the acoustic field distribution measurement results obtained for the duct crosssection located $0.51 \mathrm{~m}$ from the duct outlet edge. The acoustic pressure distribution patterns over the other two selected cross-sections, although different, presented similar picture of rms acoustic pressure dependence on the radial coordinate (distance from the duct axis $z$ ) predicted by Eq. 6 and characteristic for presence of Bessel modes.

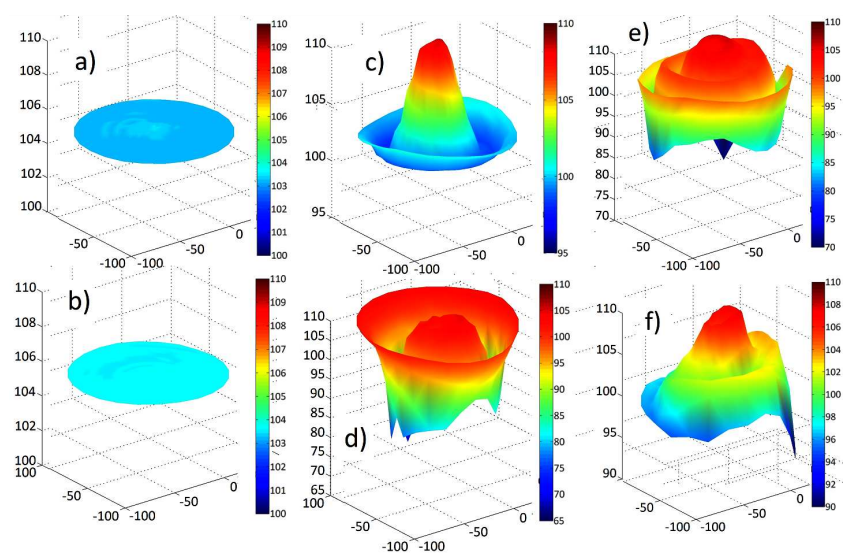

Fig. 6. The acoustic field distribution the over crosssection located $51 \mathrm{~cm}$ from the duct end: (a), (c), (e) the infinite duct model, for excitation frequencies 800 , 2850, and $5500 \mathrm{~Hz}$, respectively; (b), (d), (f) - the semi-infinite duct model, for excitation frequencies 800 , 2850 , and $5500 \mathrm{~Hz}$, respectively.

For the lowest excitation frequency, when only the plane wave generated by the source was expected to 
reach the observation point, the difference between the maximum and the minimum acoustic pressure level measured at given radial coordinate was $0.3 \mathrm{~dB}$ over the whole cross-section for both semi-infinite and infinite duct model. This is an evidence of high degree of consistence of the observed field distribution with theoretical assumptions on one hand and precision of the modified measurement setup on the other. Analyzing the acoustic field distribution pattern for $k a=2.44$ where cut-on modes includes, apart the plane wave $(0,0)$, also a nonaxisymmetric mode $(1,1)$, one can observe some deviation from axial symmetry (up to $2.2 \mathrm{~dB}$ for the infinite and $0.8 \mathrm{~dB}$ for the semi-infinite duct model) corresponding to the cosine-type dependence of the acoustic pressure in modes of that type on the azimuthal angle (cf. Eq. 6). This speaks in favor of the hypothesis providing for relatively insignificant excitation of asymmetric modes.

Therefore, one should expect certain content of asymmetric modes also in waves excited with higher frequencies. The analysis for the measured acoustic field distribution at the reduced frequency $k a=3.44$ for which the asymmetric cut-on modes include also the $(2,1)$ mode that can propagate without attenuation shows that the maximum variation of the acoustic pressure registered at the same radial coordinate and for different values of the azimuthal angle $\phi$, representing a measure of deviation from axial symmetry, amounted to $3 \mathrm{~dB}$ and $1.2 \mathrm{~dB}$ for semi-infinite and infinite duct models, respectively.

The effect of presence of asymmetric modes was observed also at the reduced frequency $k a=7.76$ for which also the $(0,2)$ mode become allowable together with asymmetric modes listed in Table. The total number of wave modes that can propagate at this frequency without attenuation is eleven, including three axisymmetric modes. For the infinite duct model, axial symmetry could be clearly seen. In the model of semi-infinite circular duct, axial symmetry was significantly affected by the above-mentioned complex diffraction phenomena occurring at the outlet (each mode was partly reflected with different amplitude change and phase shift described by means of the so-called open-end correction [16] and interfered with other reflected and transformed modes).

\section{Conclusions}

The above-described modification of the measuring setup designed for examination of acoustic field inside model hard-walled cylindrical ducts resulted in significant improvement of consistency of the obtained measurement results with theoretical predictions. In the explored frequency band $800-5500 \mathrm{~Hz}$ that for the duct inside radius of $a=0.077 \mathrm{~m}$ corresponded to the reduced frequency range $1.13<k a<7.76$, the effect of appearance of successive axisymmetric modes was observed as expected in view of the assumed axial symmetry of the system with axially mounted sound source. Although no effort was spared to maintain axial symmetry of the sys- tem undisturbed, the effect of non-axisymmetric modes could be observed for higher frequencies. The deviations from axial symmetry in acoustic pressure distribution patterns indicated presence of axially asymmetric (circumferential) modes, with the nature of these variations being consistent with the expected dependence on the azimuthal angle, especially clearly seen for $k a=4.02$.

The measurement setup presented in this paper will be subject to further modifications; in particular, measurements in duct models with much larger radii are necessary. As the number of cut-on modes depends on the reduced frequency value $k a$, examination of the same acoustic field configurations will be important at proportionally lower frequencies and longer waves that in turn is expected to reduce the acoustic field disturbance related to penetration of the duct interior with the movable microphone-carrying head and allow to achieve another level of measuring accuracy.

\section{Acknowledgments}

The investigation was supported by the Ministry of Science and Higher Education (Grant No. 2011/03/B/ST8/05042).

\section{References}

[1] E. Skudrzyk, The foundations of acoustic, SpringerVerlag, Wien 1971.

[2] H. Levine, J. Schwinger, Phys. Rev. 73, 383 (1948).

[3] G.F. Homicz, J.A. Lordi, J. Sound Vib. 41, 283 (1975).

[4] A. Snakowska, J. Phys. VI 2, 653 (1992).

[5] A. Snakowska, J. Jurkiewicz, Acta Acust./Acust. 96, 416 (2010).

[6] P. Joseph, C.L. Morfey, J. Acoust. Soc. Am. 105, 2590 (1999).

[7] J.P. Dalmont, J. Sound. Vib. 243, 441 (2001).

[8] A. Snakowska, H. Idczak, Acta Acust./Acust. 83, 955 (1997).

[9] P. Joseph, P.A. Nelson, M.J. Fisher, J. Acoust. Soc. Am. 106, 766 (1999).

[10] S. Lidoine, H. Batard, S. Troyes, A. Delnevo, M. Roger, AIAA-2001-2140AIAA/CEAS Aeroacoustics Conference and Exhib. 1, (2001).

[11] S. Sinayoko, P. Joseph, A. McAlpine, J. Acoust. Soc. Am. 127, 2159 (2010).

[12] Ł. Gorazd, J. Jurkiewicz, A. Snakowska, Archiv. Acoust. 37, 227 (2012).

[13] L. Enghardt, L. Neuhaus, C. Lowis, 10th AIAA/CEAS Aeroacoustics Conference, Manchester 2004, p. 10.

[14] J. Jurkiewicz, A. Snakowska, D. Smolik, Acta Phys. Pol. A 119, 1061 (2011).

[15] L.A. Weinstein, The theory of diffraction and the factorization method, Golem Press, Colorado 1969.

[16] A. Snakowska, J. Jurkiewicz, D. Smolik, Acta Phys. Pol. A 120, 736 (2011). 